\title{
Anakinra as a diagnostic challenge and treatment option for systemic autoinflammatory disorders of undefined etiology
}

\author{
Stephanie R. Harrison, ${ }^{1}$ Dennis McGonagle, ${ }^{1}$ Sharmin Nizam, ${ }^{2}$ Stephen Jarrett, ${ }^{2}$ \\ Jeroen van der Hilst, ${ }^{3,4}$ Michael F. McDermott, ${ }^{1}$ and Sinisa Savic ${ }^{1,5}$ \\ ${ }^{1} \mathrm{NIH}$ Research-Leeds Musculoskeletal Biomedical Research Unit (NIHR-LMBRU) and Leeds Institute of Rheumatic \\ and Musculoskeletal Medicine (LIRMM), Wellcome Trust Brenner Building, St. James's University Hospital, Beckett \\ Street, Leeds, United Kingdom. ²Department of Rheumatology, Pinderfields Hospital, Wakefield, United Kingdom. \\ ${ }^{3}$ Department of Infectious Diseases and Immunity, Jessa Hospital, Hasselt, Belgium. ${ }^{4}$ BIOMED Research Institute, \\ University of Hasselt, Hasselt, Belgium. ${ }^{5}$ Department of Clinical Immunology and Allergy, St. James's University \\ Hospital, Leeds, United Kingdom.
}

BACKGROUND. Some adult patients presenting with unexplained pyrexia, serositis, skin rashes, arthralgia, myalgia, and other symptoms commonly found in autoinflammatory disorders may not fit a specific diagnosis, either because their clinical phenotype is nondiagnostic or genetic tests are negative. We used the term undifferentiated systemic autoinflammatory disorder (USAID) to describe such cases. Given that well-defined autoinflammatory diseases show responses to IL-1 blockade, we evaluated whether anakinra was useful for both diagnosing and treating uSAID patients.

METHODS. We performed a retrospective analysis of consecutive patients presenting with uSAID between 2012-2015 who were treated with the recombinant IL-1 receptor antagonist anakinra. USAID was diagnosed after excluding malignancy, infection, and pathogenic mutations in known hereditary fever syndromes (HFS) genes and where clinical criteria for adult onset Still's disease (AOSD) were not met.

RESULTS. A total of 11 patients presented with uSAID ( 5 males and 6 females), with a mean time to diagnosis of 3.5 years (1-8 years). Patients were unresponsive or only partially controlled on disease-modifying antirheumatic drug (DMARD)/steroid treatment. Anakinra controlled symptoms within 4-6 weeks of starting treatment in 9 of 11 cases. Two patients discontinued therapy - one due to incomplete response and another due to severe injection-site reactions.

CONCLUSION. This retrospective case series demonstrates that the spectrum of poorly defined autoinflammatory disorders that show responsiveness to anakinra is considerable. Anakinra seems a viable treatment option for these patients, who are unresponsive to standard steroid/DMARD treatments. Moreover, given the mechanisms of action, response to anakinra implicates underlying IL-1 dysregulation in the disease pathogenesis of responding uSAIDs patients.

Conflict of interest: D. McGonagle received an honorarium for a lecture sponsored by SOBI. S. Savic received an educational grant and participated on advisory board meeting for SOBI.

Submitted: January 5, 2016 Accepted: March 29, 2016 Published: May 5, 2016

Reference information: JCI Insight. 2016;1(6):e86336. doi:10.1172/jci.insight.86336.

\section{Introduction}

The defining feature of systemic autoinflammatory disorders (SAIDs) is seemingly unprovoked episodic or chronic sterile inflammation that is secondary to innate immune system dysregulation $(1,2)$. These diseases clinically manifest as unexplained pyrexia, serositis, skin rashes, arthralgia, arthritis, myalgia, and other symptoms. The SAIDs comprise well-characterized monogenic forms, and to date, a number of genes have been implicated in well-defined monogenic autoinflammatory diseases (3). However, despite the recent description of new autoinflammatory disease genes and defined syndromes (4), approximately $50 \%$ of patients presenting with clinical features resembling SAIDs still do not carry any of the known germline pathogenic mutations in autoinflammatory disease genes $(5,6)$. Some of these patients will fit the 
Table 1. Demographic data and presenting signs/symptoms for all uSAID patients $(n=11)$

\begin{tabular}{|c|c|c|c|c|c|c|c|c|c|c|c|c|c|c|c|c|c|c|c|}
\hline & ஸัّ & 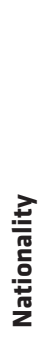 & 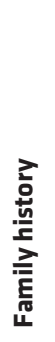 & 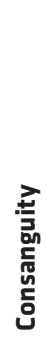 & 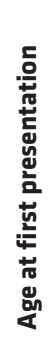 & 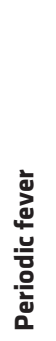 & 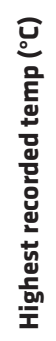 & 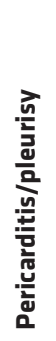 & 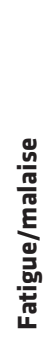 & 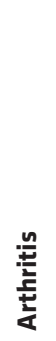 & 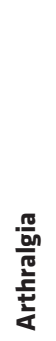 & $\frac{\frac{\pi}{a n}}{\frac{\pi}{\pi}}$ & 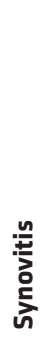 & $\begin{array}{l}\frac{5}{\text { J }} \\
\text { 凹 }\end{array}$ & 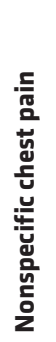 & 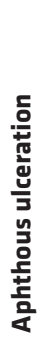 & 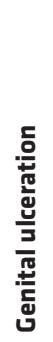 & 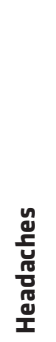 & 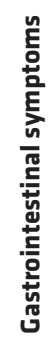 \\
\hline uSAID-01 & $M$ & B & $\mathrm{N}$ & $\mathrm{N}$ & 67 & $\mathrm{Y}$ & 41 & $\mathrm{~N}$ & $\mathrm{Y}$ & $\mathrm{Y}$ & $\mathrm{Y}$ & $Y$ & $\mathrm{Y}$ & $\mathrm{N}$ & $\mathrm{N}$ & $\mathrm{N}$ & $\mathrm{N}$ & $\mathrm{N}$ & $\mathrm{N}$ \\
\hline USAID-02 & $\mathrm{F}$ & $B$ & $\mathrm{~N}$ & $\mathrm{~N}$ & 24 & $Y$ & 41 & $Y$ & $Y$ & $Y$ & $Y$ & $Y$ & $Y$ & $Y$ & $\mathrm{~N}$ & $\mathrm{~N}$ & $\mathrm{~N}$ & $Y$ & $Y$ \\
\hline USAID-03 & $M$ & B & $\mathrm{N}$ & $\mathrm{N}$ & 34 & Y & 40 & $\mathrm{~N}$ & Y & $\mathrm{N}$ & $\mathrm{N}$ & Y & Y & $\mathrm{N}$ & $\mathrm{N}$ & $\mathrm{N}$ & $\mathrm{N}$ & $\mathrm{Y}$ & $\mathrm{N}$ \\
\hline USAID-04 & $\mathrm{F}$ & B & $\mathrm{N}$ & $\mathrm{N}$ & 24 & $\mathrm{Y}$ & 39 & $\mathrm{Y}$ & $\mathrm{Y}$ & $\mathrm{Y}$ & $\mathrm{Y}$ & $\mathrm{Y}$ & $\mathrm{Y}$ & $\mathrm{Y}$ & $\mathrm{N}$ & $\mathrm{N}$ & $\mathrm{N}$ & $\mathrm{Y}$ & $\mathrm{N}$ \\
\hline USAID-05 & $M$ & B & $\mathrm{N}$ & $\mathrm{N}$ & 32 & $\mathrm{Y}$ & $?$ & $\mathrm{~N}$ & $\mathrm{Y}$ & $Y$ & $\mathrm{Y}$ & $\mathrm{Y}$ & $\mathrm{Y}$ & $\mathrm{Y}$ & $\mathrm{N}$ & $\mathrm{N}$ & $\mathrm{N}$ & $\mathrm{N}$ & $\mathrm{N}$ \\
\hline USAID-06 & $M$ & B & $\mathrm{N}$ & $\mathrm{N}$ & 53 & $Y$ & 39 & $\mathrm{~N}$ & $Y$ & $\mathrm{~N}$ & $Y$ & $\mathrm{~N}$ & $\mathrm{~N}$ & $\mathrm{Y}$ & $\mathrm{N}$ & $\mathrm{N}$ & $\mathrm{N}$ & $Y$ & $Y$ \\
\hline uSAID-10 & $M$ & B & $\mathrm{N}$ & $\mathrm{N}$ & 76 & $\mathrm{Y}$ & 40 & $\mathrm{~N}$ & $\mathrm{Y}$ & $\mathrm{N}$ & $\mathrm{Y}$ & $\mathrm{Y}$ & $\mathrm{N}$ & $\mathrm{Y}$ & $\mathrm{N}$ & $\mathrm{N}$ & $\mathrm{N}$ & $\mathrm{N}$ & $\mathrm{N}$ \\
\hline USAID-11 & $\mathrm{F}$ & B & $\mathrm{N}$ & $\mathrm{N}$ & 35 & $\mathrm{Y}$ & $?$ & $\mathrm{~N}$ & $\mathrm{Y}$ & $\mathrm{N}$ & $\mathrm{N}$ & $\mathrm{N}$ & $\mathrm{N}$ & $\mathrm{N}$ & $\mathrm{N}$ & $\mathrm{Y}$ & $\mathrm{N}$ & $\mathrm{Y}$ & $\mathrm{N}$ \\
\hline
\end{tabular}

The demographic data and presenting signs/symptoms for all uSAIDs patients ( $n=11$. B, British; F, female; I, Israel; M, male; N, no; Y, yes; ?, unknown.

criteria for less well-characterized polygenic forms of autoinflammatory diseases, such as adult onset Still's disease (AOSD) (7). However, a proportion of adult patients will have variable expression of clinical signs and symptoms and, thus, do not necessarily fulfill all clinical criteria for diagnosis of AOSD. Therefore, we use the term undifferentiated SAID (uSAID) to identify this subset of patients who fail to meet criteria for the established monogenic or polygenic autoinflammatory diseases but seem to have clinical features in keeping with an autoinflammatory disorder.

Currently, there are no diagnostic criteria or tests for uSAID patients and no laboratory investigations to definitively distinguish sterile inflammatory responses, caused by dysregulation of the innate immune system, from inflammatory responses provoked by other causes, such as infection. Furthermore, diagnosis of polygenic autoinflammatory diseases, some of which even have defined clinical criteria such as AOSD, is in any case challenging. Therefore, recognizing uSAID requires an even higher degree of clinical suspicion and, like other conditions in this disease area, remains a diagnosis of exclusion. Consequently, patients are often subject to multiple invasive investigations, thereby delaying treatment, which, in any event, is suboptimal with existing strategies and which typically includes nonspecific immunosuppression with corticosteroids and other disease-modifying antirheumatic drugs (DMARDs).

Several of the monogenic SAIDs, especially cryopyrin associated periodic syndromes (CAPS), have genetic dysregulation of IL-1 $\beta$ and IL-18 pathways, with associated increased proinflammatory cytokine release (5). The cryopyrinopathies show rapid responses to IL-1 antagonism, with sustained efficacy (812). Remarkably, several other monogenic autoinflammatory disorders — not necessarily directly linked to underlying NLRP3 inflammasome dysregulation, including TNF receptor-associated periodic syndrome (TRAPS) (13) - also show a response to IL-1 antagonists, as do poorly defined polygenic autoinflammatory disorders, including AOSD (7).

Consequently, we reasoned that uSAIDs might show robust responses to anakinra (Kineret) - a short acting recombinant IL-1 receptor antagonist - and, thus, could be used as a rapid diagnostic tool for the stratification and treatment of uSAIDs with underlying IL-1 dysregulation. We used this empirical approach in our routine clinical practice to select patients who might benefit from treatment with anakinra. Herein, we describe 11 consecutive patients presenting with clinical features of SAID who did not meet clinical criteria for diagnosis of AOSD, as proposed by Yamaguchi et al. (14), and in whom anakinra therapy was generally informative for diagnostic confirmation and treatment of the underlying condition. 
Table 2. uSAID patients' biochemical profiles, investigations, and treatments $(n=11)$

\begin{tabular}{|c|c|c|c|c|c|c|c|c|c|c|c|c|c|c|c|c|c|c|c|c|c|}
\hline & \multirow[b]{2}{*}{ 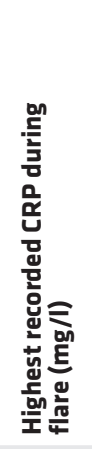 } & \multicolumn{4}{|c|}{ Biochemical profile } & \multirow[b]{2}{*}{ 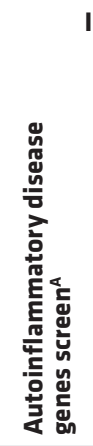 } & \multicolumn{7}{|c|}{$\begin{array}{l}\text { Investigations required to exclude } \\
\text { alternative diagnoses }\end{array}$} & \multirow[b]{2}{*}{ 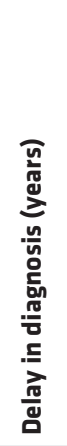 } & \multicolumn{4}{|c|}{ Failed treatments } & \multirow[b]{2}{*}{ 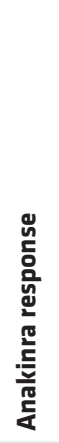 } & \multirow[b]{2}{*}{ 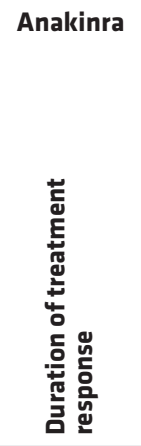 } & \multirow[b]{2}{*}{ 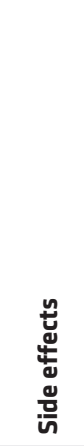 } \\
\hline & & 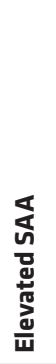 & 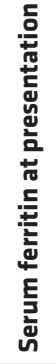 & 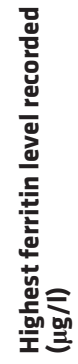 & 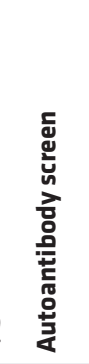 & & 七! & 与 & $\bar{\Sigma}$ & 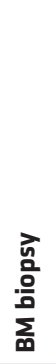 & 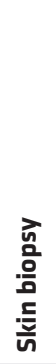 & 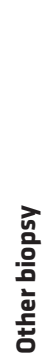 & 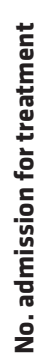 & & 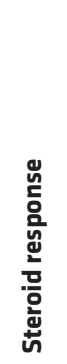 & 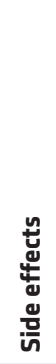 & 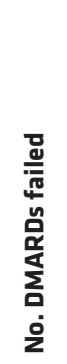 & 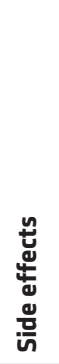 & & & \\
\hline USAID-01 & 217.0 & $\mathrm{Y}$ & $\mathrm{N}$ & 440 & -ve & Y & $\mathrm{Y}$ & $\mathrm{Y}$ & $\mathrm{N}$ & $\mathrm{N}$ & $\mathrm{N}$ & $\mathrm{N}$ & 2 & 3 & $P$ & $\mathrm{~N}$ & 1 & $\mathrm{~N}$ & $\mathrm{Y}$ & $\begin{array}{l}1.4 \text { yrs - } \\
\text { ongoing }\end{array}$ & $\mathrm{N}$ \\
\hline uSAID-02 & 40.0 & $\mathrm{Y}$ & $\mathrm{N}$ & $\mathrm{N}$ & APLA & Y & $\mathrm{N}$ & $\mathrm{Y}$ & $\mathrm{N}$ & $\mathrm{N}$ & $\mathrm{N}$ & $\mathrm{N}$ & 1 & 5 & $\mathrm{~N}$ & $\mathrm{~N}$ & $1^{\mathrm{B}}$ & $\mathrm{N}$ & $\mathrm{Y}$ & $\begin{array}{l}1 \mathrm{yr}- \\
\text { ongoing }\end{array}$ & $\mathrm{N}$ \\
\hline USAID-05 & 182.0 & $?$ & $\mathrm{~N}$ & $\mathrm{~N}$ & -ve & Y & $\mathrm{N}$ & $\mathrm{N}$ & Y & $\mathrm{N}$ & $N$ & $\mathrm{~N}$ & 3 & 1 & $P$ & $\mathrm{Y}$ & $2^{\mathrm{B}}$ & $\mathrm{Y}$ & $\mathrm{Y}$ & $\begin{array}{l}1.8 \text { yrs - } \\
\text { ongoing }\end{array}$ & $\mathrm{N}$ \\
\hline USAID-06 & 106.0 & $\mathrm{Y}$ & $\mathrm{N}$ & 324 & -ve & Y & $\mathrm{Y}$ & $\mathrm{Y}$ & $\mathrm{N}$ & $\mathrm{Y}$ & $\mathrm{Y}$ & $\mathrm{Y}$ & 1 & 2 & $P$ & $\mathrm{Y}$ & 3 & $\mathrm{Y}$ & $\mathrm{N}$ & $\begin{array}{l}12 \text { weeks } \\
\text { total }\end{array}$ & Severe \\
\hline USAID-07 & 44.0 & $\mathrm{Y}$ & $\mathrm{N}$ & $\mathrm{N}$ & -ve & Y & $\mathrm{Y}$ & $\mathrm{Y}$ & $\mathrm{N}$ & $\mathrm{N}$ & $\mathrm{N}$ & $N$ & 2 & 1 & $\mathrm{n} / \mathrm{a}$ & $N$ & $\mathrm{n} / \mathrm{a}$ & $\mathrm{N}$ & $\mathrm{Y}$ & $\begin{array}{l}1.2 \text { yrs - } \\
\text { ongoing }\end{array}$ & $\mathrm{N}$ \\
\hline USAID-08 & 260.0 & $?$ & $\mathrm{~N}$ & $\mathrm{~N}$ & -ve & Y & $\mathrm{N}$ & $\mathrm{Y}$ & $\mathrm{N}$ & $\mathrm{N}$ & $\mathrm{N}$ & Y & 1 & 2 & $P$ & $\mathrm{~N}$ & 2 & $\mathrm{~N}$ & Y & $\begin{array}{l}6.2 \text { yrs - } \\
\text { ongoing }\end{array}$ & $\mathrm{N}$ \\
\hline USAID-09 & 177.0 & $?$ & $\mathrm{~N}$ & $\mathrm{~N}$ & -ve & Y & $Y$ & $\mathrm{Y}$ & $\mathrm{N}$ & Y & Y & $\mathrm{Y}$ & 1 & 1 & $\mathrm{~N}$ & $\mathrm{~N}$ & 2 & $\mathrm{Y}$ & Y & $\begin{array}{c}8 \mathrm{~m}- \\
\text { deceased }\end{array}$ & $\mathrm{N}$ \\
\hline USAID-10 & 237.0 & Y & $\mathrm{N}$ & 600 & -ve & Y & $\mathrm{Y}$ & $\mathrm{N}$ & $\mathrm{Y}$ & $\mathrm{N}$ & $N$ & $N$ & 6 & 7 & $\mathrm{~N}$ & $\mathrm{~N}$ & $1^{*}$ & $\mathrm{Y}$ & $\mathrm{Y}$ & $\begin{array}{c}3 \mathrm{~m}- \\
\text { deceased }\end{array}$ & $\mathrm{N}$ \\
\hline
\end{tabular}

uSAIDs patients' biochemical profiles, investigations, and treatments $(n=11)$. Autoantibody screen was according to standard practice and included screening for RF, ACPA, ANA, ANCA, and antiphospholipid (APLA) antibodies. Other biopsies included video-assisted thorascopic surgery (VATS), biopsy, liver, and kidney. ${ }^{A}$ Autoinflammatory disease gene panel included MEFV, NLRP3, TNFRSF1A, and NOD2. ${ }^{B}$ These patients had failed colchicine treatment. 'Due to funding restrictions, we were unable to increase the dose of anakinra for this patient; therefore, treatment had to be discontinued due to there only being evidence of partial response. CT, computerized tomography; DMARDs, disease-modifying antirheumatic drugs; MRI, magnetic resonance imaging; n/a, not applicable; N, no; P, partial response; Y, yes; -ve, negative; ?, unknown.

\section{Results}

uSAID patients presented with a range of nonspecific autoinflammatory symptoms including periodic fevers (11 of 11), fatigue/malaise (11 of 11), rash (8 of 11), myalgia (9 of 11), arthralgia (8 of 11), synovitis (6 of 11), arthritis (5 of 11), headaches (5 of 11), lymphoreticular (4 of 11) as well as gastrointestinal symptoms ( 3 of 11) and pericarditis/pleurisy ( 2 of 11) (Table 1). The average age of symptom onset was 43.7 years (range 24-76), and there was no family history of autoinflammatory disease or consanguinity.

All patients experienced substantial delays between presentation and eventual diagnosis (mean 2.9 years [1.0-8.0]) requiring input from various other specialties, including hematology, infectious diseases, urology, respiratory, neurorehabilitation, and dermatology. In addition, uSAID patients were subjected to extensive investigation, including a full autoantibody screen, serum ferritin, CT (9 of 11), PET-CT (8 of 11), biopsies (skin/BM/liver/ kidney/lymph nodes) (6 of 11) and magnetic resonance imaging (MRI) (2 of 11) (Table 2), all of which failed to establish an underlying cause. Furthermore, uSAID patients tested negative for known autoinflammatory disease genes (NLRP3, MEFV, TNFRSF1A, and NLRC2 [NOD2]) (11 of 11). During the course of these investigations, management with nonspecific immunosuppressive agents (a combination of NSAIDs, DMARDs, and systemic corticosteroids) failed to control autoinflam- 


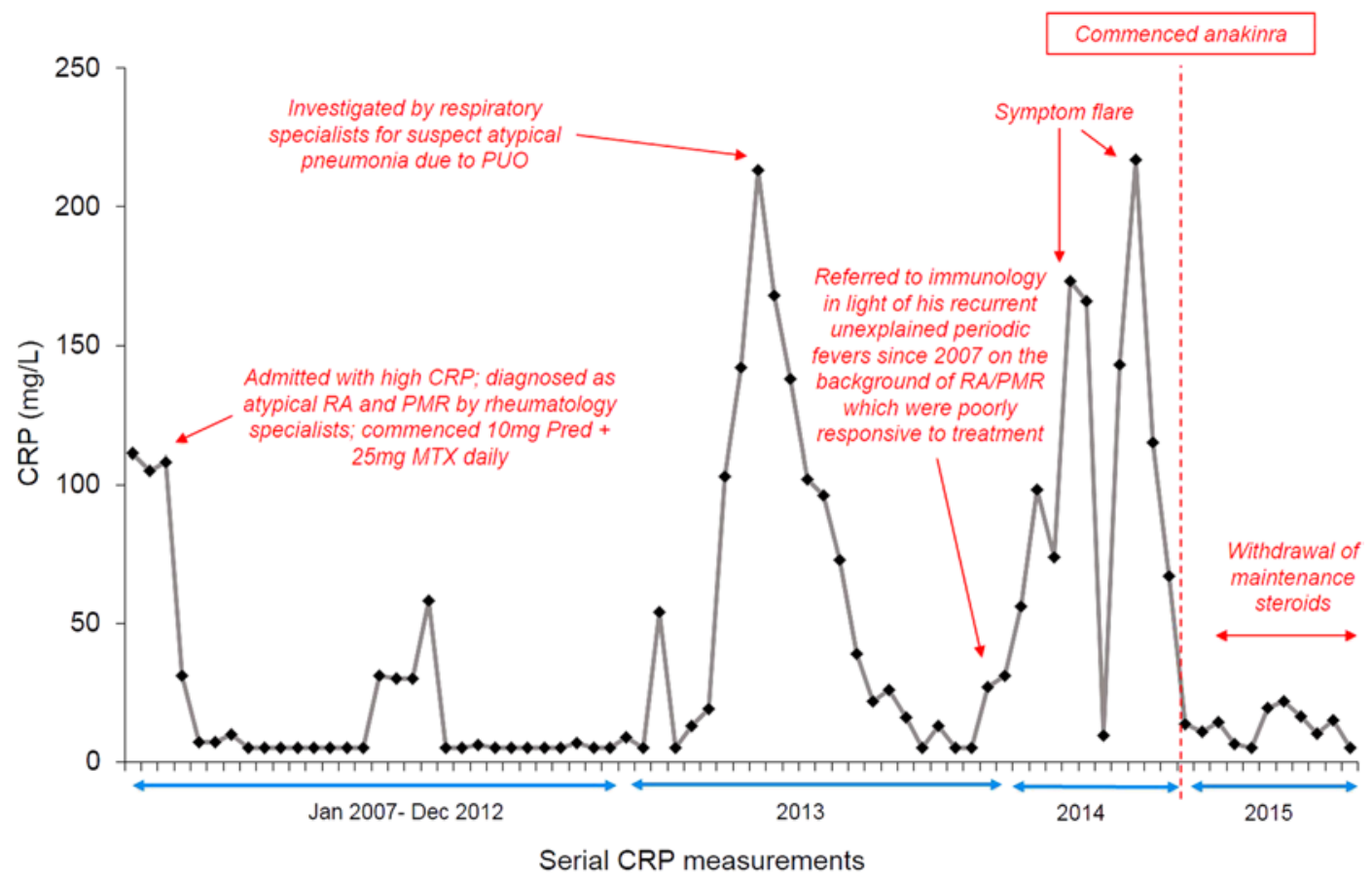

Figure 1. uSAID-01: serial CRP measurements. This figure shows the serial CRP measurements recorded for uSAID-01 from initial presentation to Dec. 2015. CRP, C-reactive protein; PMR, polymyalgia rheumatica; Pred, prednisolone; PUO, pyrexia of unknown origin; RA, rheumatoid arthritis; MTX, methotrexate.

matory symptoms. Systemic corticosteroid treatment was ineffective in 5 of 11 patients and only partially controlled symptoms in 6 of 11 patients, of which 3 of 11 experienced substantial adverse effects from prolonged steroid use, including intolerable weight gain and diabetes. In addition, 4 of 11 patients experienced adverse events as a consequence of DMARD treatment (Table 2).

Contrastingly, anakinra produced full symptom control in 9 of 11 patients within 4-6 weeks of starting treatment (Table 2), which correlated with a clinically marked reduction in C-reactive protein (CRP), eventually enabling these patients to gradually withdraw concomitant corticosteroids and/or DMARD treatment. Anakinra was generally well tolerated. The treatment was discontinued in 2 patients only; in one due to an incomplete response and, in another, due to a marked adverse event, namely injection-site reactions. Two patients died during the follow-up period. Patient uSAID-10 died due to complications of influenza, which were not thought to be directly related to his treatment with anakinra. Patient uSAID-09 died from what was thought to be a progression of her underlying disease. A more detailed description of all cases is given below.

USAID-01. uSAID-01 is a 74-year-old male who initially presented with an 8-year history of fatigue, malaise, arthritis, arthralgia, myalgia, and elevated CRP and serum amyloid A (SAA). Neutrophils and ferritin were within the reference range. Following further investigations, including autoimmune screening, a diagnosis of seronegative rheumatoid arthritis (RA) was made and methotrexate (MTX) plus corticosteroids commenced. Subsequently, the patient developed shoulder pain - in addition to sacroiliac and axial joint pain, jaw stiffness, and overwhelming fatigue - and was diagnosed with polymyalgia rheumatica (PMR). In 2013, uSAID-01 was admitted with a suspected PMR flare. Upon further questioning, it was noted that he had, in fact, experienced periodic fevers for several years. Despite having ongoing joint symptoms for over 8 years and seemingly poor disease control on MTX, he did not develop any joint erosions. Furthermore, uSAID-01's PMR had an unusually severe systemic component and was only partially responsive to corticosteroids (the highest dose of prednisolone used was $60 \mathrm{mg}$ daily orally [PO] and maintenance dose $10 \mathrm{mg}$ daily PO), despite PMR being characteristically highly corticosteroid responsive. A PET-CT showed increased tracer activity in multiple joints, suggesting active arthropathy but showing no evidence of vasculitis or malignancy. Considering that some of his clinical features were atypical of seronegative RA and PMR, and as he had disproportionately elevated CRP (>100 $\mathrm{mg} / \mathrm{ml})$, the patient was given an empirical trial of anakinra, $100 \mathrm{mg}$ daily s.c. This successfully controlled all symptoms, substan- 


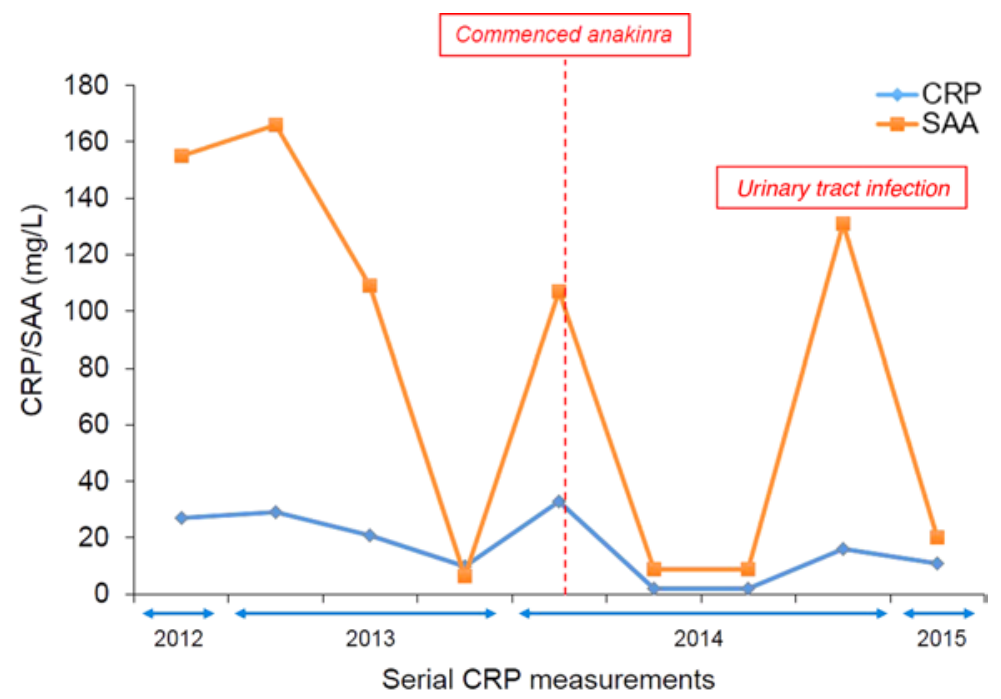

Figure 2. uSAID-02: serial CRP and SAA measurements. This figure shows the serial CRP/SAA measurements recorded for uSAID-02 from initial presentation to Dec. 2015. CRP, C-reactive protein; SAA, serum amyloid A.

tially alleviating the fatigue and malaise, and resulted in reduction of CRP (Figure 1). The patient has now successfully stopped cortiocosteroids without a flare of his symptoms.

USAID-02. uSAID-02 is a 39-year-old lady who presented at age of 26 with monthly periodic fever episodes, accompanied by fatigue, malaise, headaches, drenching night sweats, nausea, leg cramps, and occasional vomiting. Her CRP and SAA were markedly elevated during the attacks and would normalize when asymptomatic. Her serum ferritin levels remained within the reference at all times. Initially, her symptoms improved during pregnancy; however, following delivery, her attacks increased in frequency and she developed a generalized, blotchy, pruritic papular rash. She had extensive investigations and - apart from a positive lupus anticoagulant screen, which was not thought to be relevant to her symptoms - all other tests were negative (Table 2). uSAID-02 did not respond to maintenance prednisolone PO and was only partially responsive to high-dose methylprednisolone $(80 \mathrm{mg})$ and $40 \mathrm{mg}$ prednisolone $\mathrm{PO}$ during a flare. Therefore, uSAID-02 was commenced on $100 \mathrm{mg}$ daily s.c. anakinra, following which she experienced only occasional mild residual breakthrough symptoms that were effectively managed with an extra dose of anakinra. Over time, uSAID-02's response to anakinra greatly improved, and daily maintenance with anakinra alone is currently sufficient to achieve complete symptomatic control (Figure 2).

USAID-03. uSAID-03 is a 35-year-old gentleman who presented following admission to hospital with a 1-year history of pyrexia of unknown origin (PUO), weight loss, fatigue, and elevated CRP. uSAID-03 had a history of foreign travel; however, a complete infectious disease screen was negative. His other investigations showed normal wbc and elevated platelets (highest recoded platelet count was 520: range 150-400 $\times 10^{9} / 1$ ) and normal serum ferritin. His symptoms initially resolved following a course of high-dose oral corticosteroids (prednisolone $40 \mathrm{mg}$ daily PO); however, the patient was readmitted 2 months later with a repeat febrile episode. Subsequently, the patient has continued to experience intermittent fevers (3-4 weekly lasting 24-48 hours), fatigue, joint swelling, and headaches. His symptoms were partially responsive to corticosteroids (15 mg prednisolone daily PO); however, uSAID-03 experienced intolerable weight gain as a result. MTX also failed to control break-through symptoms, necessitating admission to hospital for treatment and leaving the patient unable to work. Treatment with $100 \mathrm{mg}$ daily s.c. anakinra partially controlled symptoms, with a decreased frequency of fever episodes and improved energy levels, as well as reduced CRP. However, CRP remained mildly elevated despite doubling the daily dose of anakinra, and the patient continued to be corticosteroid dependent. Furthermore, the patient developed a blistering skin rash with erythema at the injection site. Therefore, he was switched to tocilizumab (Actemra). His symptoms are now well controlled, his CRP has normalized, and he has now discontinued all corticosteroids (Figure 3).

uSAID-04. uSAID-04 is a 39-year-old lady with an 8-year history of periodic fevers, headaches, polyarthralgia, a widespread urticarial rash, and elevated CRP that developed after her first pregnancy and delivery, which were uncomplicated. Ferritin and neutrophil counts were normal. Symptoms were partially 


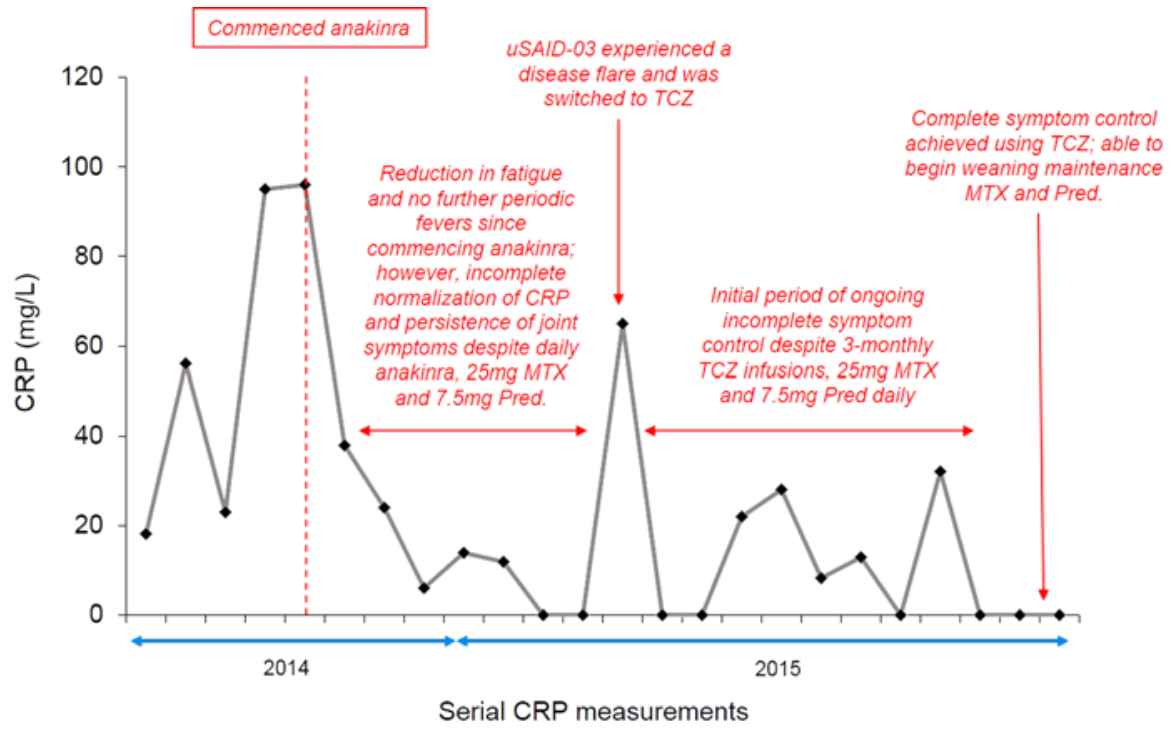

Figure 3. uSAID-03: serial CRP measurements. This figure shows the serial CRP measurements recorded for uSAID-03 from initial presentation to Dec. 2015. CRP, C-reactive protein; MTX, methotrexate; Pred, prednisolone; TCZ, tocilizumab.

responsive to corticosteroid depot injections and resolved within 2 years. However, during her third pregnancy, she relapsed, experiencing episodic fevers, polyarthralgia, synovitis, overwhelming fatigue, pericardial symptoms, and headaches, and she also developed a nonpainful, nonpruritic, generalized macular erythematous rash. CRP and SAA were both elevated at this time. PET-CT was negative, and skin biopsy showed a mild sprinkling of chronic inflammatory cells in perivascular areas and no obvious evidence of vasculitis, granulomatous inflammation, or malignancy, which was deemed to be essentially inconclusive. MTX failed to control symptoms, leaving uSAID-04 corticosteroid dependent (15-20 mg maintenance prednisolone daily PO) with incomplete control of her symptoms; however, $100 \mathrm{mg}$ daily s.c. anakinra completely resolved symptoms and enabled her to gradually reduce and stop her corticosteroids. USAID-04 attempted to reduce the frequency of her anakinra injections to once every 2 days, but this resulted in a flare of her underlying condition. However, upon recommencing her initial anakinra $100 \mathrm{mg}$ daily regimen, uSAID-04 returned to being symptom free with well-controlled CRP levels (Figure 4).

USAID-05. uSAID-05 is a 43-year-old male who presented with periodic fevers (2-3 episodes annually) accompanied by lower back and shoulder girdle pain with elevated CRP. Ferritin and neutrophil counts were normal. Initial investigations ruled out recurrent pyelonephritis; however, we found no clear cause of his symptoms, which persisted and led to hospital admission on 3 occasions. He later developed oligoarthritis, tenosynovitis, mild swelling of his right hand and wrist, myalgia, moderate morning stiffness, and a macular erythematous rash (left flank and right abdomen). He was intolerant of azathioprine and only partially responsive to mycophenolate mofetil (MMF) and $10 \mathrm{mg}$ daily maintenance prednisolone PO. Over time, he also developed a Cushingoid appearance, associated with the long-term corticosteroid use; however, we were unable to reduce his prednisolone without precipitating a flare of his symptoms. Treatment with $100 \mathrm{mg}$ daily s.c. anakinra completely controlled his symptoms, enabling him to eventually completely withdraw his maintenance prednisolone and MMF. His disease remains fully controlled with daily injections of anakinra alone (Figure 5).

USAID-06. uSAID-06 is a 53-year-old gentleman who presented with a 2-year history of fever, flu-like symptoms, widespread intermittent nonpruritic papular erythematous rash, night sweats, arthralgia, headaches, and elevated CRP. His initial investigations revealed lymphopenia $\left(0.34 \times 10^{9} / 1\right)$, with normal neutrophil and platelet count and haemoglobin levels. He also had normal urea and electrolytes (U\&Es), liver function tests (LFTs), and serum ferritin levels. Extended viral screen was negative for HIV and hepatitis A, B, and C, and there was no evidence of acute EBV or CMV reactivation. The skin biopsy showed moderate perivascular chronic inflammatory cell infiltrate within the dermis, predominantly composed of lymphocytes, and there was no evidence of fibrinoid necrosis or malignancy. Urticarial vasculitis was suggested as a provisional diagnosis, and small vessel vaculitis was also considered as one of the differential diagnoses; however, his autoimmune screen 


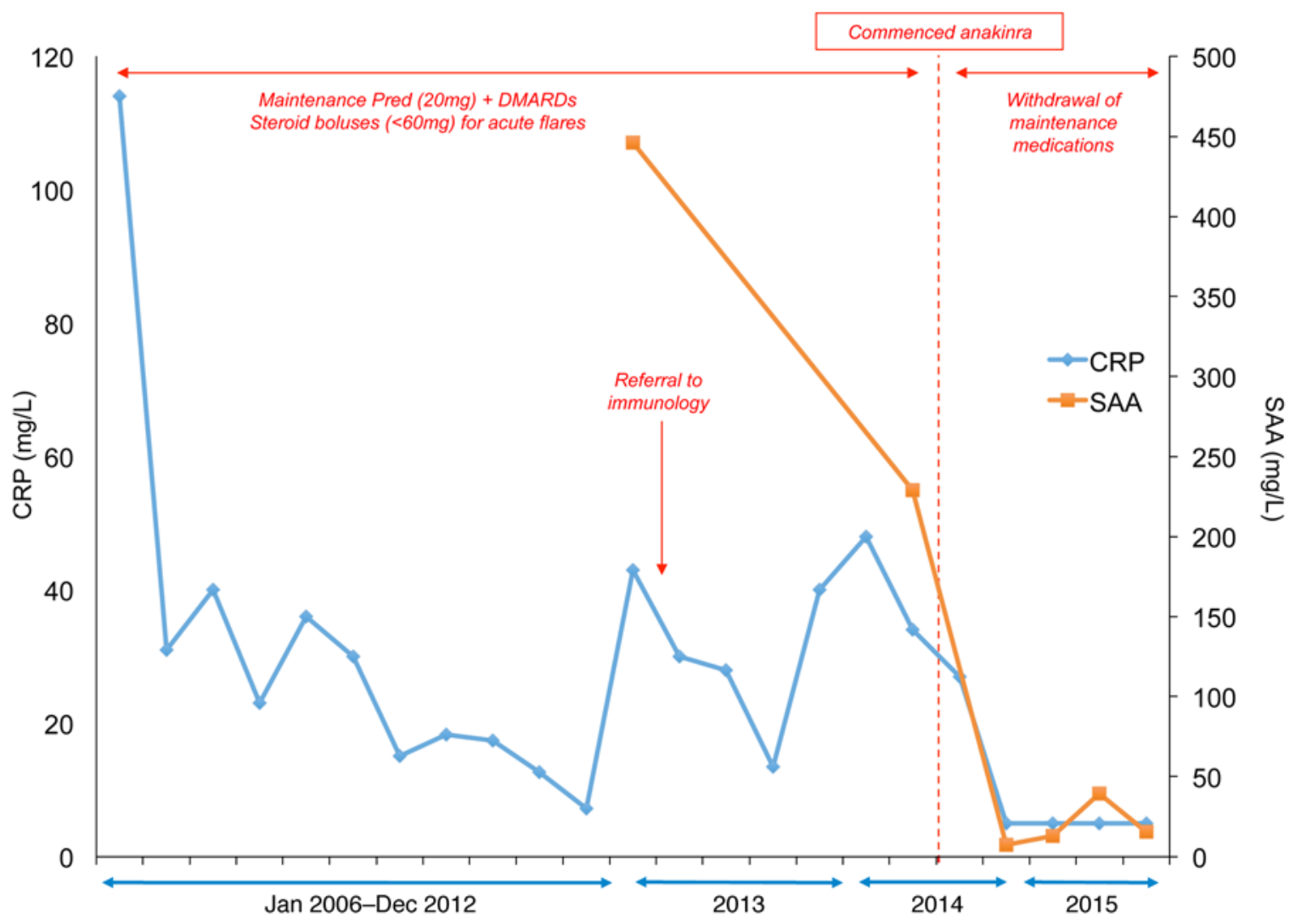

Figure 4. uSAID-04: serial CRP and SAA measurements. This figure shows the serial CRP/SAA measurements recorded for uSAID-04 from initial presentation to Dec. 2015. CRP, C-reactive protein; DMARDs, disease-modifying antirheumatic drugs; Pred, prednisolone; SAA, serum amyloid A.

was negative, and a CT of his sinuses and thorax was normal. uSAID-06 was initially treated with prednisolone, starting with a dose of $40 \mathrm{mg}$ daily PO. Although he did respond to this, the response was only partial; he became corticosteroid dependent (20-30 mg of prednisolone daily PO) and developed corticosteroid-induced glaucoma and a Cushingoid appearance. His symptoms were also unresponsive to MTX, and the patient experienced adverse reactions to azathioprine and MMF, which exacerbated his underlying inflammatory condition. Therefore, $100 \mathrm{mg}$ daily s.c. anakinra commenced; he experienced improvement in his symptoms and was able to reduce corticosteroids to $5 \mathrm{mg}$ of prednisolone daily from a previous 20-mg maintenance dose. However, he developed a severe injection-site reaction with a painful blistering skin rash, and treatment was therefore withdrawn. The rash resolved without scarring after a few weeks; the patient was subsequently switched to tocilizumab (Actemra), to which he has shown a favorable response (Figure 6).

uSAID-07. uSAID-07 is a 33-year-old lady of Israeli-Jewish ancestry who presented with PUO, fatigue, and a 1-month history of sore throat. Four months previously, she had developed a transient circular rash on her left thigh. Her sore throat resolved spontaneously; however, she continued to experience profound fatigue - with episodes of pyrexia lasting a few days — that resolved spontaneously and presented with persistently elevated CRP. Her other investigations showed normal wbc, marginally elevated platelet count (the highest recoded was $420 \times 10^{9} / 1$ : range $150-400 \times 10^{\%} / 1$ ), normal serum ferritin, and elevated SAA. Subsequently, she developed transient left knee and shoulder joint arthralgia and synovitis, which resolved completely within 48 hours after taking ibuprofen. Her genetic investigations identified an NLRP3 variant of unknown significance (Q703K) that not thought to be pathogenic. She was commenced on $100 \mathrm{mg}$ daily s.c. anakinra, following which her symptoms improved and was associated with persistent normalization of CRP (Figure 7).

USAID-08. uSAID-08 is a 67-year-old lady who presented with a 2-year history of frequent attacks of episodic fever, sweats, arthralgia, and grossly elevated CRP. Serum ferritin and neutrophil counts were normal at the time of the initial presentation. She was initially treated with corticosteroids and MTX, which failed to control her symptoms. Her CRP normalized, and her symptoms resolved after starting $100 \mathrm{mg}$ daily s.c. anakinra. Failure to take anakinra precipitated a flare on 2 separate occasions; one required admission to 


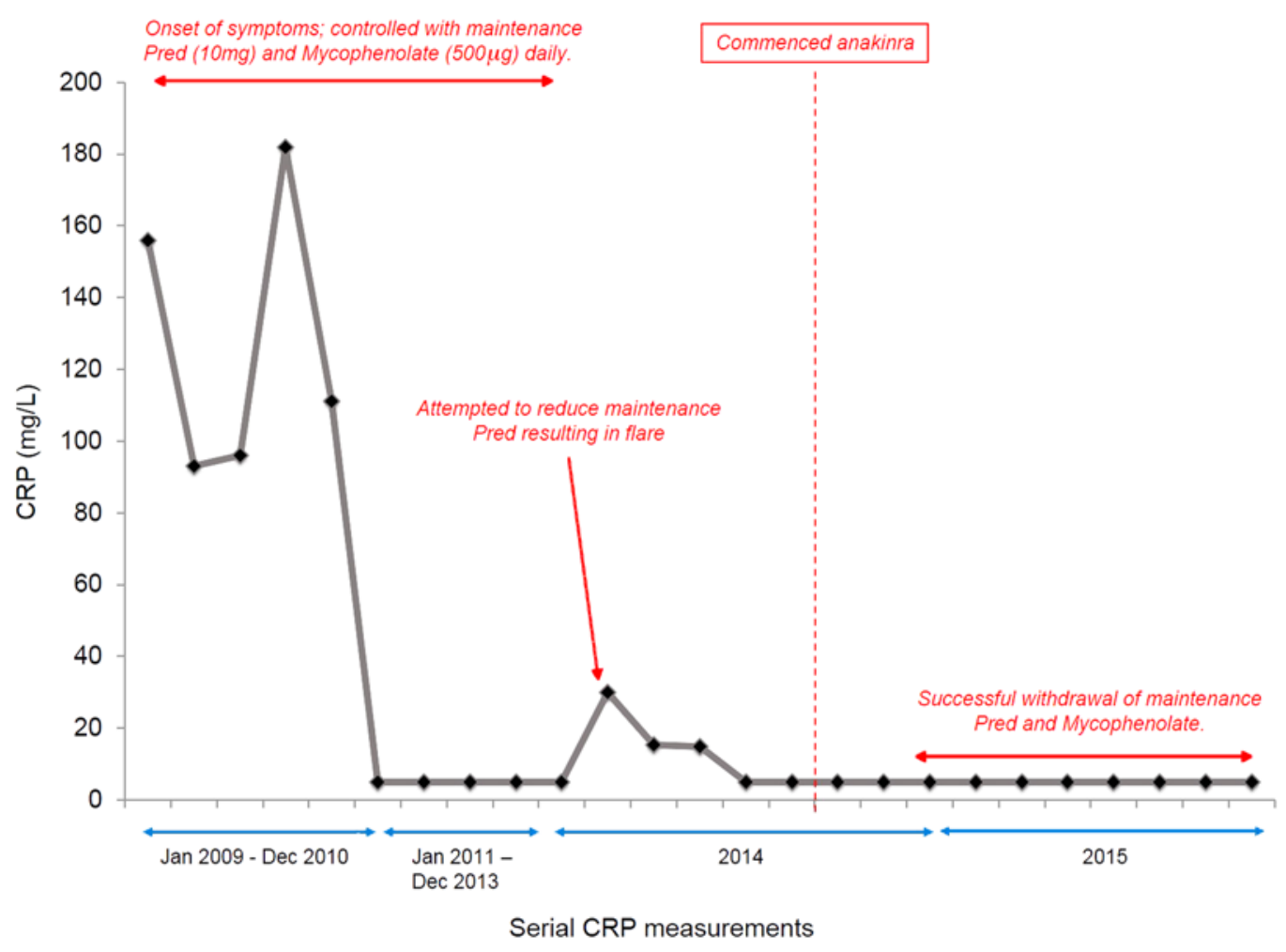

Figure 5. uSAID-05: serial CRP measurements. This figure shows the serial CRP measurements recorded for uSAID-05 from initial presentation to Dec. 2015. CRP, C-reactive protein; Pred, prednisolone.

an intensive treatment unit (ITU), where the patient was found to have grossly elevated LFTs, raising suspicions of possible hemophagocytic syndrome. However, CT and liver biopsy were both inconclusive, and her LFTs spontaneously recovered. Despite maintenance of anakinra, some of her symptoms increased in severity, with increased frequency of episodic night sweats, aphthous ulceration, and a pruritic skin rash. These were initially managed with high-dose corticosteroids; however, management of subsequent flares with an increased dose of anakinra proved extremely effective, leading to long-term remission of disease (Figure 8).

USAID-09. uSAID-09 is a 42-year-old female who presented with a 2-year history of sore throat, widespread skin rash, lymphadenopathy, orogenital ulceration, and elevated CRP. Ferritin levels and neutrophil counts were all within the normal range, and a screen for autoantibodies was also negative. Whole body CT showed widespread lymphadenopathy, but multiple biopsies including BM, lymph nodes, skin, and liver showed reactive changes only and no evidence of malignancy. The autoimmune screen was negative, and serum ferritin was within normal limits. The skin rash was eventually diagnosed as pityriasis rubris pilaris. The patient was partially responsive to $20 \mathrm{mg}$ oral prednisolone and was subsequently started on cyclosporine $(2 \mathrm{mg} / \mathrm{kg})$, which she had difficulty tolerating. Unfortunately, after a few weeks of treatment, and after having an additional single dose of MTX (7.5 mg), she developed pneumocystis jiroveci pneumonia, requiring ITU admission. Her LFT's became briefly abnormal during this period but improved after cyclosporin and MTX were stopped. Subsequently, she remained dependent on high-dose corticosteroids (up to $40 \mathrm{mg}$ of prednisolone daily PO) to control her skin rash and fevers. Following introduction of anakinra, her CRP normalized and corticosteroids were gradually tapered without flare of her symptoms (Figure 9). She remained on treatment with anakinra for a period of 8 months, during which time she remained generally well and was able to reduce prednisolone to $2 \mathrm{mg}$ daily. Unfortunately, her condition worsened, and she again became unwell, with progressive lymphadenopathy, respiratory symptoms, and fevers. She was admitted to ITU, where no clear cause for her deterioration was found. She died of multisystem organ failure shortly afterward. Postmortem examination findings were largely inconclusive, and - although atypical $\mathrm{T}$ cell lymphoma was suggested as a differential diagnosis — skin, lymph node, and BM biopsies conducted during the postmortem examination failed to find objective evidence of changes consistent with $\mathrm{T}$ cell lymphoma. 


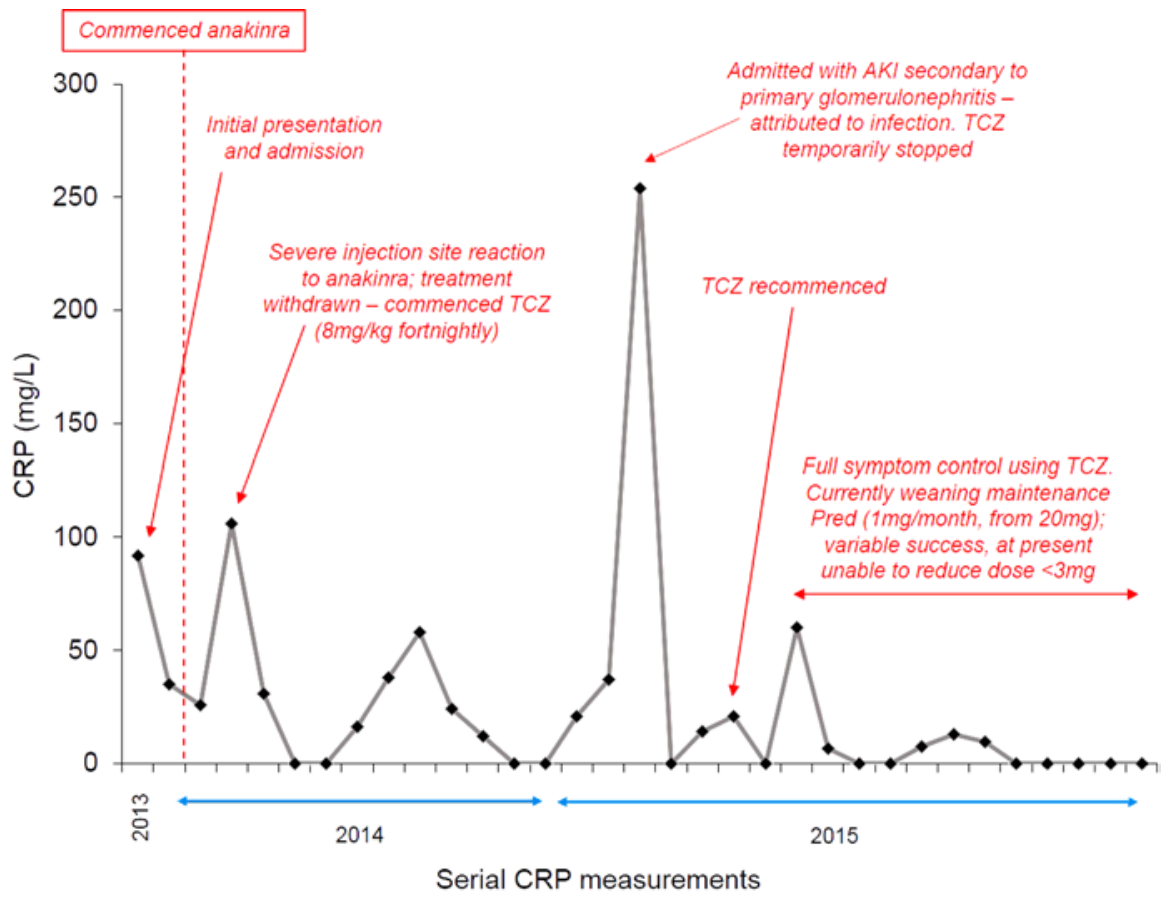

Figure 6. uSAID-06: serial CRP measurements. This figure shows the serial CRP measurements recorded for uSAID-06 from initial presentation to Dec. 2015. CRP, C-reactive protein; Pred, prednisolone; TCZ, tocilizumab; AKI, acute kidney injury.

USAID-10. uSAID-10, an 81-year-old male, presented with fever, rigors, fatigue/malaise, and dermatitis with persistently elevated CRP. He also had a complex medical history, including chronic unexplained anemia, and was previously assessed for possible PMR. Initial investigations revealed narrowing of the ureter, raising the suspicion of transitional cell carcinoma; however, this was excluded following further urological tests. Over the next 4 years, further extensive investigations failed to establish the cause of uSAID-10's symptoms, which continued to be poorly controlled, and he required hospital admission on 7 separate occasions. This patient's symptoms were refractory to oral corticosteroids (15 mg prednisolone daily PO) and MTX; however, $100 \mathrm{mg}$ daily s.c. anakinra produced complete symptom control with only residual fatigue, and he was able to begin tapering his maintenance prednisolone from $15 \mathrm{mg}$ to $7.5 \mathrm{mg}$ daily. Unfortunately, a few months after commencing anakinra, the patient was admitted with seasonal flu, which was complicated by pneumonia. Although he initially did improve with antibiotics, he subsequently suffered a fatal myocardial infarction (Figure 10).

uSAID-11. A 35-year-old female presented 4 weeks after giving birth to her second child. She experienced severe abdominal pain with spiking fever. The fever was accompanied by cervical lymphadenopathy, mild aphthous stomatitis, headache, and malaise. At that point, she experienced similar attacks of 3-5 days durations every 4-6 weeks. Extensive investigations yielded no diagnosis, but it showed elevated CRP during episodes of fever. Because of the FMF-like phenotype, colchicine was initiated, but this had no effect on the frequency or severity of attacks. There was no clinical response to high-dose methylprednisolone $(1 \mathrm{mg} / \mathrm{kg})$, started at the onset of an attack. Three years after onset, the frequency of her attacks increased to every 2-3 weeks. The severity of attacks was disabling, and the patient was unable to perform her job. Eventually, a trial of anakinra was started. At the first signs of an attack, the patient self-injected $100 \mathrm{mg}$ anakinra s.c. This led to fever resolution within hours, with relief from abdominal pain; cessation of anakinra resulted in disease flare. The patient was able to taper the dose to 5 injections per week and was able to restart her full-time job as a teacher. Currently, she uses anakinra approximately 5 days per month.

\section{Discussion}

Our understanding of the breadth and complexity of monogenic diseases that span the immunological disease continuum (IDC) is continuously expanding - some of these conditions may respond fully to IL-1 blockade and others to a lesser extent or not at all (15). The autoinflammatory disease category was first proposed in 1999 to encompass some of the distinct clinicopathological features of a group of monogenic conditions, 


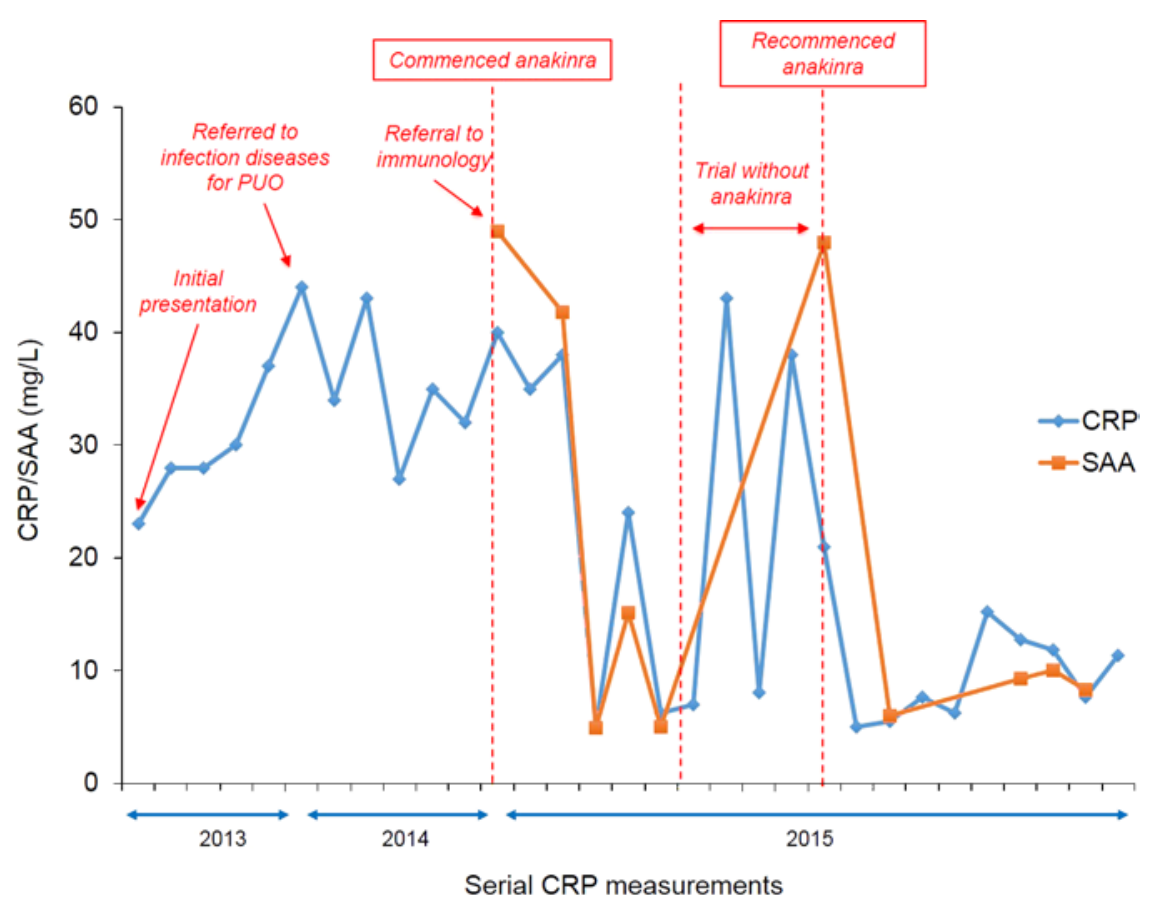

Figure 7. uSAID-07: serial CRP and SAA measurements. This figure shows the serial CRP and SAA measurements recorded for uSAID-07 from initial presentation to Dec. 2015. CRP, C-reactive protein; PUO, pyrexia of unknown origin; SAA, serum amyloid A.

characterized by recurrent episodes of inflammation, without high-titer autoantibodies or antigen-specific $\mathrm{T}$ cells (16). It was later proposed that the majority of inflammatory disorders are situated along the IDC, with genetic disorders of innate and adaptive immunity located at either end of the spectrum, and that the former may respond to proinflammatory cytokine blockade (17). At the molecular level, IL-1 dysregulation is a common pathological feature of many prototypical autoinflammatory disorders such as hereditary fever syndromes (HFS) (5, 8-12). The classical IL-1 family of cytokines, including IL-1 $\alpha$ and IL-1 $\beta$, as well as IL-18, are central players in the inflammatory response, which incorporates induction of constitutional symptoms, such as fever, malaise, myalgia, stimulation of acute phase responses resulting in recruitment of neutrophils, and induction of other proinflammatory cytokines, such as IL-6 and TNF (18). Whereas in the case of HFS, inappropriate IL-1 release is linked to dysregulated NLRP3 inflammasome, this is less clear for polygenic autoinflammatory disorders. However, there is evidence that, in addition to NLRP3 inflammasome-generated mature IL-1 $\beta$, the pro-IL-1 $\beta$ form may also be cleaved outside the cells; mature IL- $1 \beta$ is virtually absent inside cells, and caspase 1 is actively secreted outside the cell (19). Other proteases, apart from caspase 1, present in the inflammatory extracellular fluid play important roles in cleavage and activation of IL-1 $\beta$, and these alternative activation mechanisms may account for a significant proportion of biologically active IL-1 $\beta$ (19). This might explain why anti-IL-1 therapy is an effective treatment for a number of other disorders that are thought to be at least partly autoinflammatory in nature; for example, systemic juvenile idiopathic arthritis (sJIA) (20), idiopathic recurrent pericarditis (21), and Behçet's disease (22). In addition, similar IL-1 dysregulation may complicate other pathological conditions, including primary immunodeficiency $(4,23)$ and malignancy (24). An example of the latter is Schnitzler's syndrome, which is a SAID associated with paraproteinemia that frequently precedes the development of frank hematological malignancy (24).

The findings presented here strongly suggest that a response to anakinra is a valuable diagnostic tool and effective therapy for uSAID, thereby implicating an underlying IL-1 dysregulation in these seemingly heterogeneous cases. The short half-life of anakinra, and the rapid and sustained nature of the responses observed, enabled the rapid differentiation of responding from nonresponding patients. Furthermore, it has minimized the exposure of nonresponding patients to unnecessary immunosuppression when compared with other DMARDs or biological treatments, such as the IL-6 receptor antagonist tocilizumab, which was used as second-line treatment in the 2 nonresponding patients. Moreover, the short half-life of anakinra also minimizes the duration of adverse effects related to treatment, should they develop, as was the case for uSAID-06. 


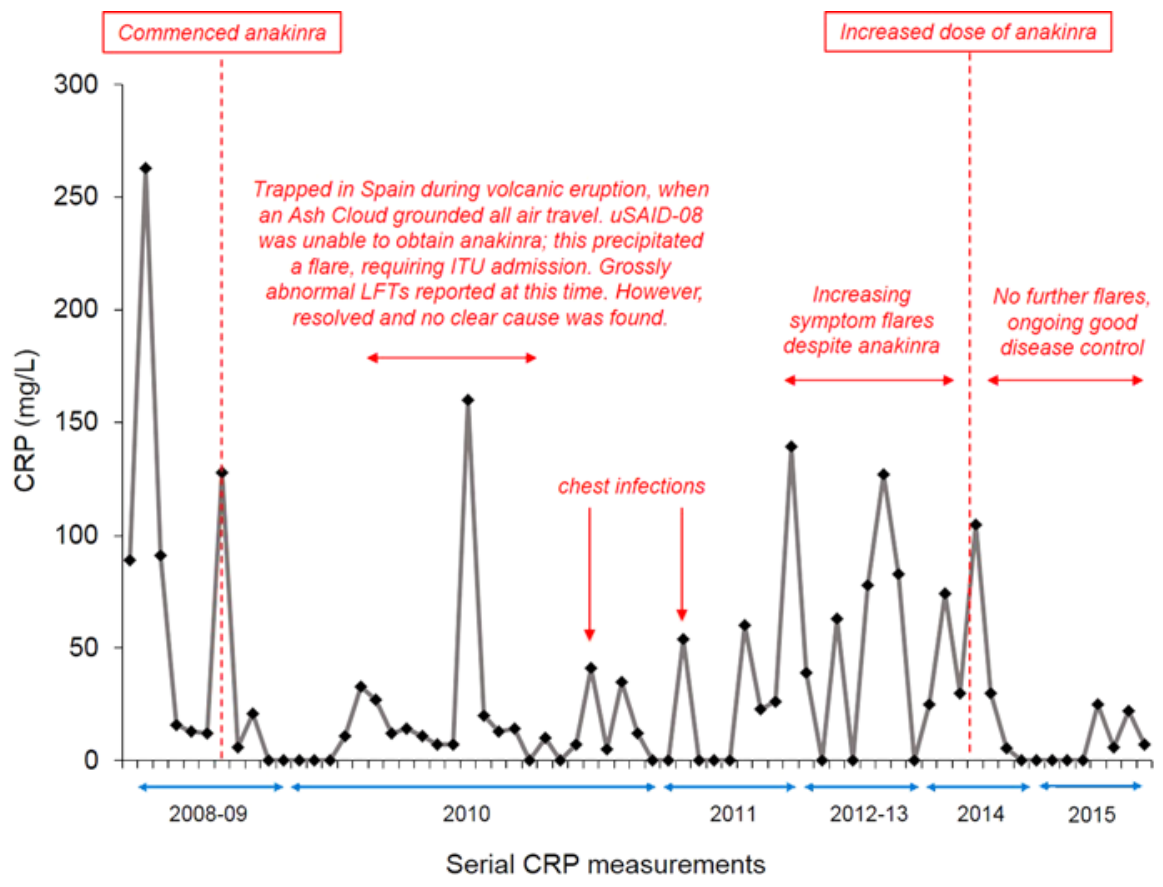

Figure 8. uSAID-08: serial CRP measurements. This figure shows the serial CRP measurements recorded for uSAID-08 from initial presentation to Dec. 2015. CRP, C-reactive protein; Pred, prednisolone; TCZ, tocilizumab.

It is reasonable to argue that some of the cases labeled USAID and described here would, over time, progress to fulfill criteria for a clinically defined SAID, such as AOSD, which is now widely considered to be a polygenic autoinflammatory condition (25). However, despite several classification criteria for AOSD, it is still a relatively poorly defined entity (7). This is illustrated by the fact that no biological marker, including ferritin, is thought to be uniquely specific for this condition (7). However, anakinra is recommended for the treatment of $\operatorname{AOSD}(7,26)$, and therefore, empirical use of anakinra would still result in early implementation of appropriate treatment for such patients.

There are several reasons why uSAID might be a useful term to employ in everyday clinical practice. It allows clinicians to start to define some of the cases, which may present with vague and overlapping clinical features but which might have an autoinflammatory etiology. This term implies that the diagnosis needs to be frequently revisited, as some cases will inevitably progress and evolve into a more defined clinical entity. Therefore, clinicians might be inclined to review such patients on a more regular basis and look for changes in signs and symptoms that might suggest the presence of a different disease etiology. Furthermore, a label of uSAID can be easily revised if additional information should become available. For example, wider application of massive parallel sequencing has led to the ongoing discovery of germline mutations, which will be relevant in some of these cases. In addition, somatic NLRP3 mutations have recently been described in adult patients, resulting in CAPS phenotype (27). Such mutations would not be picked up using traditional sequencing methods such as Sanger sequencing of selected targeted genes, which were used for investigation of cases we presented here. However, a good therapeutic response to anakinra would be a clue to look further for such pathology, and therefore, using anakinra as a diagnostic test would still make sense.

Although this retrospective case series is focused on anakinra as the treatment regime of choice, it would be interesting to see if similar effects were produced with canakinumab, a longer acting IL-1 $\beta-$ specific antagonist that does not neutralize the IL-1 $\alpha$ cytokine. Furthermore, it is possible that higher doses of IL-1 antagonism may be required to induce a clinical response in some patients who we have classified as partial responders, such as uSAID-03. However, due to funding restrictions, we were unable to implement higher doses than $200 \mathrm{mg}$ daily of anakinra in our clinic, despite our intention to do so. There also remains a question of what role is played by other proinflammatory cytokines such as IL- 6 and TNF in this process and whether it would be possible, using additional clinical and laboratory biomarkers, to select patients for appropriate therapy without prior therapeutic trial. Nonetheless, this 


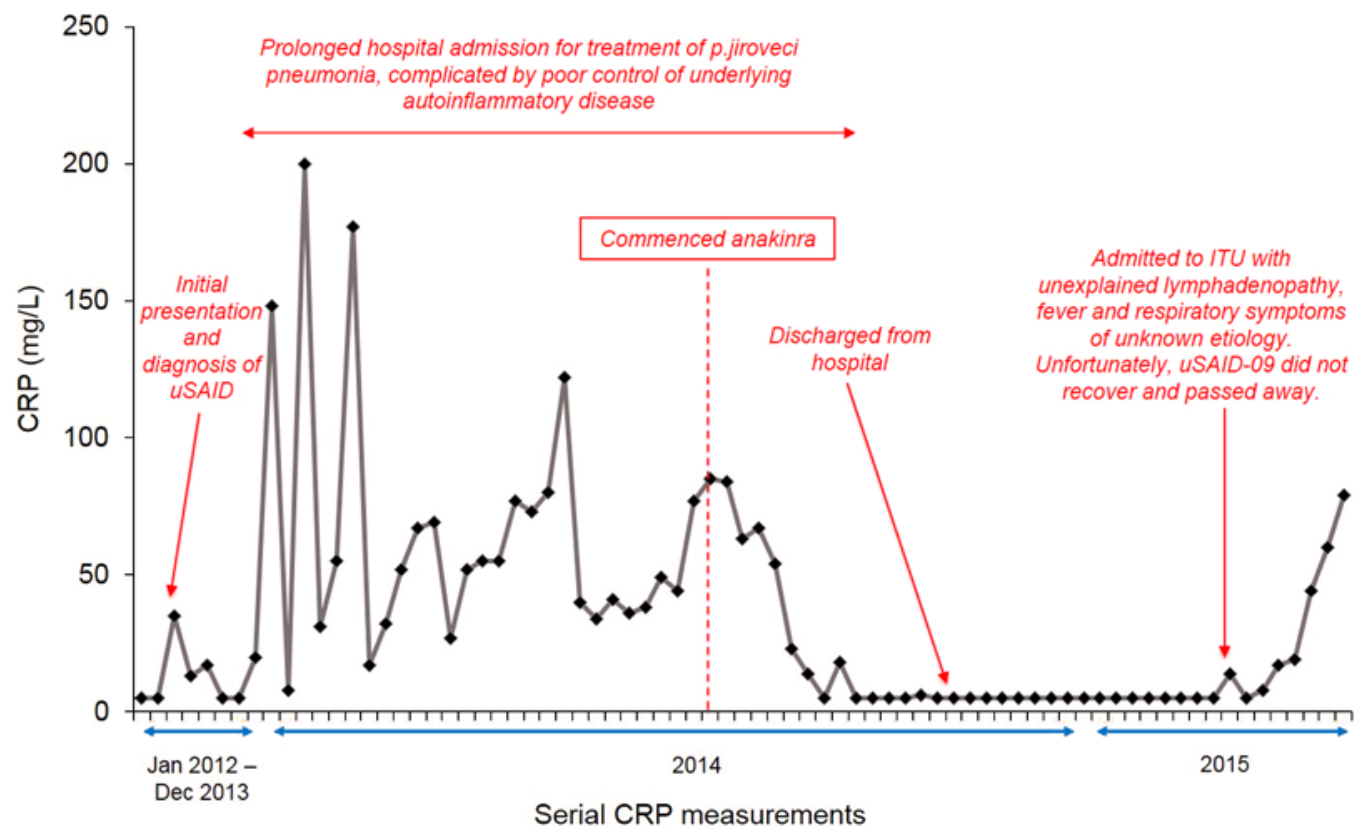

Figure 9. uSAID-09: serial CRP measurements. This figure shows the serial CRP measurements recorded for uSAID-09 from initial presentation in 2012 until mortality in April 2015. CRP, C-reactive protein; ITU, intensive treatment unit; p.jiroveci pneumonia, pneumocystis jiroveci pneumonia (an aggressive atypical form of pneumonia).

case series of patients strongly suggests that a clinical response to anakinra could be an effective diagnostic and treatment strategy for uSAID. Formal evaluation of these findings is now being undertaken in a larger cohort of patients.

\section{Methods}

Basic demographic and clinical data, collected as part of standard care, were used to conduct a retrospective case series that evaluated the outcomes of 10 patients ( 5 male and 5 female) presenting to a multidisciplinary specialist clinic for SAID, based at Leeds Teaching Hospital from 2012-2015, and 1 female patient, who presented to the Department of Infectious Diseases and Immunity.

Structure of the clinic. In 2012, a specialist immunology autoinflammatory clinic was established in the Leeds Teaching Hospitals NHS Trust and University of Leeds. The hospital and university network serve as a tertiary referral center for 4.6 million people in the Yorkshire, England, region. In conjunction with the academic Rheumatology Department at the University of Leeds, which has a longstanding interest in the diagnosis and classification of autoinflammatory disease, the local consultants and regional body of rheumatologists, dermatologists, and immunologists were informed. Difficult cases - presenting with clinical features suggestive of autoinflammatory disease, as described below, and with high acute phase responses that did not settle on therapy or were diagnostically challenging - were sought. The clinic runs on a monthly basis, and 5-6 patients are typically seen per clinic. This includes new and follow-up patients, with both genetically or clinically defined SAID, and patients with uSAID. The patients are seen jointly by a consultant immunologist and rheumatologist, with a specialist interest in SAID. The majority of patients who were initially seen in this clinic were patients with genetically or clinically defined SAID, such as periodic fever syndrome (PFS) or ASOD. However, as the service has gradually become more visible, the number of referrals has increased, as has the complexity of cases that are seen. Currently, about $50 \%$ of referrals are patients who are labeled as uSAID.

Referral pathway. Most referrals are form secondary care centers, including general rheumatology clinics, infectious diseases, hematology, and dermatology.

Patient assessment. The majority of patients have had detailed diagnostic work-up prior to referral to this service. Typically, this would include extensive infectious, malignant, and autoimmune screening. The standard infectious screen includes viral serology (hepatitis A, B, and C; HIV; CMV; and EBV), bacterial, and, where indicated, mycobacterial blood cultures. If clinically indicated, an additional 


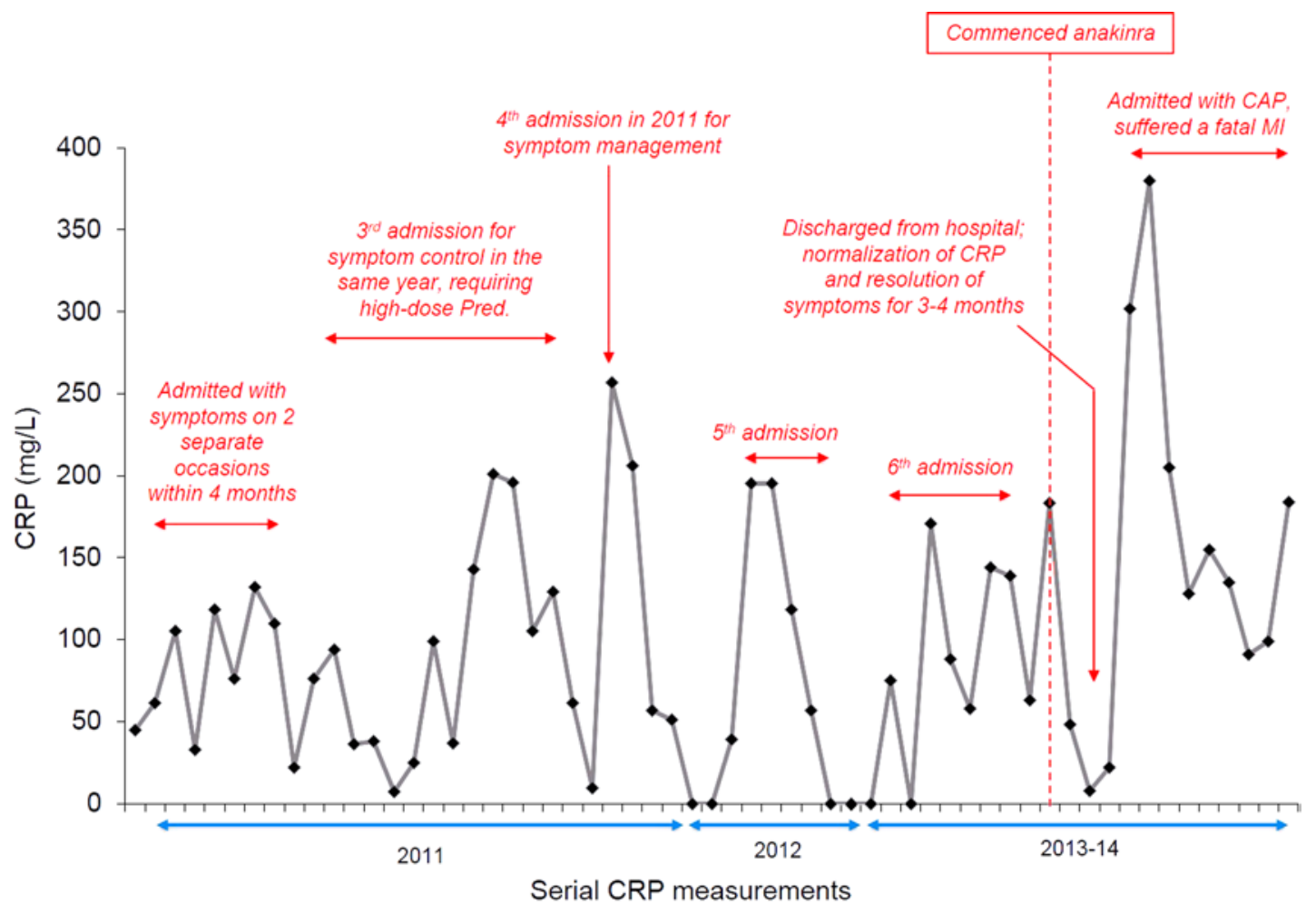

Figure 10. uSAID-10: serial CRP measurements. This figure shows the serial CRP measurements recorded for uSAID-10 from initial presentation until mortality in April 2014. CAP, community acquired pneumonia; CRP, C-reactive protein; MI, myocardial infarction; Pred, prednisolone.

infectious screen is performed, usually following a consultation with the infectious disease team. Similarly, depending on specific clinical circumstances, the patients undergo screening for malignancy using diagnostic imaging, such as whole body CT or PET-CT, and, where indicated, biopsies of relevant tissues. The results of these investigations are reviewed with the patients' clinical history. Depending on the available information, some patients are asked to keep symptom diaries and have additional CRP and SAA measurements, taken during and in between flares of the disease. The patients were assigned the term uSAID when the following conditions were met. Firstly, they had to be experiencing clinical features to suggest underlying autoinflammatory disease, as outlined by McDermott et al., that could not be explained by autoimmune, infectious, or malignant processes (16). Typically, patients have episodic or persistent constitutional symptoms, including fever, malaise, and/or weight loss. These could be associated with more organ-specific manifestations, such as sore throat, skin rashes, serositis, arthralgia, myalgia, lymphadenopathy, and other symptoms that are indicative of a dysregulated innate immune response. Secondly, there must be an absence of pathogenic mutations in known autoinflammatory disease genes and failure to meet the diagnostic criteria for defined polygenic autoinflammatory conditions, particularly AOSD. Subsequent to a diagnosis of uSAID being made, all patients in this series were treated with anakinra. The criteria agreed with a funding body to use anakinra in selected cases, including a clinical diagnosis of SAID in a patient who has severe disabling symptoms that are inadequately controlled by DMARDs and/or who is at risk of developing significant toxicity from their current therapy, for example corticosteroids. These patients gave informed verbal consent; however, formal ethical approval was not required, since this was a retrospective case series that reported outcomes of standard clinical practice.

Laboratory assessment of inflammatory markers. This included erythrocyte sedimentation rate (ESR), CRP, and SAA levels. ESR was measured using the Westergren method ( $\mathrm{mm} /$ hour) and was considered normal if $<15 \mathrm{~mm} /$ hour for males and $<20 \mathrm{~mm} /$ hour for females. Serum CRP concentrations were measured using a nephelometric immunoassay $(\mathrm{mg} / \mathrm{dl})$; values $<0.5 \mathrm{mg} / \mathrm{dl}$ were considered normal. SAA levels were measured by particle-enhanced nephelometry (BNII autoanalyzer; Dade Behring, Siemens). Reference value $<6.4 \mathrm{mg} / 1$. 
Genetic testing was undertaken by a national reference laboratory. This testing was performed, using Sanger sequencing and focusing on selected exomes, to potentially detect over $85 \%$ of all mutations found in NLRP3, TNFRSF1A, and MEFV genes.

\section{Author contributions}

SS, MFM, DM, and SRH designed the study. SRH, SS, SJ, SN, and JVDH collected the clinical information.

\section{Acknowledgments}

The authors would like to thank the Leeds Teaching Hospitals Charitable Trustees (9/R21/14-07) and the NIHR-Leeds Musculoskeletal Biomedical Research Unit that funded S. Savic, D. McGonagle, and M.F. McDermott, and the Wolfson Foundation that funded an intercalated degree research scholarship for S.R. Harrison. We also thank all clinicians involved in care of these patients. Finally, we would like to thank our patients.

Address correspondence to: Sinisa Savic, Department of Clinical Immunology and Allergy, National Institute for Health Research-Leeds Musculoskeletal Biomedical Research Unit (NIHR-LMBRU) and Leeds Institute of Rheumatic and Musculoskeletal Medicine (LIRMM), Wellcome Trust Brenner Building, St. James University, Beckett Street, Leeds, LS9 7TF, United Kingdom. Phone: 441132065567; E-mail: s.savic@leeds.ac.uk.

1. Savic S, Dickie LJ, Wittmann M, McDermott MF. Autoinflammatory syndromes and cellular responses to stress: pathophysiology, diagnosis and new treatment perspectives. Best Pract Res Clin Rheumatol. 2012;26(4):505-533.

2. Lachmann HJ. Clinical immunology review series: An approach to the patient with a periodic fever syndrome. Clin Exp Immunol. 2011;165(3):301-309.

3. Martinon F, Aksentijevich I. New players driving inflammation in monogenic autoinflammatory diseases. Nat Rev Rheumatol. 2015;11(1):11-20.

4. Aksentijevich I. Update on genetics and pathogenesis of autoinflammatory diseases: the last 2 years. Semin Immunopathol. 2015;37(4):395-401.

5. Rigante D, Vitale A, Lucherini OM, Cantarini L. The hereditary autoinflammatory disorders uncovered. Autoimmun Rev. 2014;13(9):892-900

6. Cantarini L, et al. The labyrinth of autoinflammatory disorders: a snapshot on the activity of a third-level center in Italy. Clin Rheumatol. 2015;34(1):17-28.

7. Mahroum N, Mahagna H, Amital H. Diagnosis and classification of adult Still's disease. J Autoimmun. 2014;48-49:34-37.

8. Koné-Paut I, Galeotti C. Anakinra for cryopyrin-associated periodic syndrome. Exp Rev Clin Immunol. 2014;10(1):7-18.

9. Hoffman HM, et al. Prevention of cold-associated acute inflammation in familial cold autoinflammatory syndrome by interleukin-1 receptor antagonist. Lancet. 2004;364(9447):1779-1785.

10. Goldbach-Mansky R, et al. Neonatal-onset multisystem inflammatory disease responsive to interleukin-1 $\beta$ inhibition. $N$ Engl $J$ Med. 2006;355(6):581-592.

11. Hoffman HM, et al. Efficacy and safety of rilonacept (interleukin-1 Trap) in patients with cryopyrin-associated periodic syndromes: results from two sequential placebo-controlled studies. Arthritis Rheum. 2008;58(8):2443-2452.

12. Lachmann HJ, et al. Use of canakinumab in the cryopyrin-associated periodic syndrome. N Engl J Med. 2009;360(23):24162425 .

13. Gattorno M, et al. Persistent efficacy of anakinra in patients with tumor necrosis factor receptor-associated periodic syndrome. Arthritis Rheum. 2008;58(5):1516-1520.

14. Yamaguchi M, et al. Preliminary criteria for classification of adult Still's disease. J Rheumatol. 1992;19(3):424-430.

15. Savic S, McDermott MF. Clinical genetics in 2014: New monogenic diseases spanning the immunological disease continuum. Nat Rev Rheumatol. 2014;11(2):67-68.

16. McDermott MF, et al. Germline mutations in the extracellular domains of the $55 \mathrm{kDa}$ TNF receptor, TNFR1, define a family of dominantly inherited autoinflammatory syndromes. Cell. 1999;97(1):133-144.

17. McGonagle D, McDermott MF. A proposed classification of the immunological diseases. PLoS Med. $2006 ; 3(8):$ e297.

18. Dinarello CA. A clinical perspective of IL-1 $\beta$ as the gatekeeper of inflammation. Eur J Immunol. 2011;41(5):1203-1217.

19. Wittmann M, Kingsbury SR, McDermott MF. Is caspase 1 central to activation of interleukin-1? Joint Bone Spine. 2011;78(4):327-330.

20. Adamopoulos IE. Autoimmune or autoinflammatory? Bad to the bone. Int J Clin Rheumatol. 2015;10(1):5-7.

21. Finetti M, et al. Long-term efficacy of interleukin-1 receptor antagonist (anakinra) in corticosteroid-dependent and colchicine-resistant recurrent pericarditis. J Pediatr. 2014;164(6):1425-1431.

22. Emmi G, et al. Efficacy and safety profile of anti-interleukin-1 treatment in Behçet's disease: a multicenter retrospective study. [published online ahead of print July 10, 2015]. Clin Rheumatol. doi:10.1007/s10067-015-3004-0.

23. Giannelou A, Zhou Q, Kastner DL. When less is more: primary immunodeficiency with an autoinflammatory kick. Curr Opin Allergy Clin Immunol. 2014;14(6):491-500.

24. Sokumbi O, Drage LA, Peters MS. Clinical and histopathologic review of Schnitzler syndrome: The Mayo Clinic experience 
(1972-2011). J Am Acad Dermatol. 2012;67(6):1289-1295.

25. Efthimiou P, Moorthy LN, Mavragani CP, Skokos D, Fautrel B. Adult onset Still's disease and autoinflammation. Int J Inflam. 2012;2012:964751.

26. Hong D, Yang Z, Han S, Liang X, Ma K, Zhang X. Interleukin 1 inhibition with anakinra in adult-onset Still disease: a meta-analysis of its efficacy and safety. Drug Des Devel Ther. 2014;8:2345-2357.

27. Zhou Q, et al. Brief report: cryopyrin-associated periodic syndrome caused by a myeloid-restricted somatic NLRP3 mutation. Arthritis Rheumatol. 2015;67(9):2482-2486. 